\title{
HEAT TRANSFER OF VISCOELASTIC FLUID FLOW DUE TO NON- LINEAR STRETCHING SHEET WITH INTERNAL HEAT SOURCE
}

\author{
M.M. NANDEPPANAVAR ${ }^{*}$ \\ Department of PG and UG Studies in Mathematics \\ Government College \\ Gulbarga-585105, Karnataka, INDIA \\ E-mail: mahantesh_maths@yahoo.co.in \\ M.N. SIDDALINGAPPA \\ Department of Mathematics \\ Gulbarga University \\ Gulbarga, Karnataka, INDIA \\ H. JYOTI \\ Department of Mathematics \\ Singhania University \\ Pachauri Bari-333515, Rajasthan, INDIA
}

\begin{abstract}
In the present paper, a viscoelastic boundary layer flow and heat transfer over an exponentially stretching continuous sheet in the presence of a heat source/sink has been examined. Loss of energy due to viscous dissipation of the non-Newtonian fluid has been taken into account in this study. Approximate analytical local similar solutions of the highly non-linear momentum equation are obtained for velocity distribution by transforming the equation into Riccati-type and then solving this sequentially. Accuracy of the zero-order analytical solutions for the stream function and velocity are verified by numerical solutions obtained by employing the Runge-Kutta fourth order method involving shooting. Similarity solutions of the temperature equation for non-isothermal boundary conditions are obtained in the form of confluent hypergeometric functions. The effect of various physical parameters on the local skin-friction coefficient and heat transfer characteristics are discussed in detail. It is seen that the rate of heat transfer from the stretching sheet to the fluid can be controlled by suitably choosing the values of the Prandtl number $\operatorname{Pr}$ and local Eckert number E, local viscioelastic parameter $k_{l}^{*}$ and local heat source/ sink parameter $\beta^{*}$.
\end{abstract}

Key words: viscoelastic fluid, boundary layer flow, exponential stretching sheet, heat source/sink, heat transfer and skin friction.

\section{Introduction}

Pioneering works of Sakiadis (1961) who initiated the study of the boundary layer flow over a continuous solid surface moving with constant speed and Crane (1970) on a stretching sheet have led to a great deal of work on various aspects of momentum and heat transfer characteristics in a viscoelastic boundary layer second order fluid flow over a stretching plastic boundary (Rajagopal et al., 1984; 1987), (Troy et al., 1987), (Dandapat and Gupta, 1989), (Chang, 1989), (Rollins and Vajravelu, 1991), (Andersson, 1992), (Lawrence and Rao, 1992), (Char, 1994). Dandapat et al. (1994) studied such flows and concluded that fluid elasticity has a stabilizing influence on the flow provided the wavelength of the disturbances does not exceed the viscoelastic length scale. Some of the typical applications of such study are polymer sheet

\footnotetext{
* To whom correspondence should be addressed
} 
extrusion from a dye, glass fiber and paper production, drawing of plastic films etc. In this process the sheets are drawn through a static fluid with a controlled cooling system. Extensive literature is also available including those cited above on the two-dimensional viscoelastic boundary layer flow over a stretching surface where the velocity of the stretching surface is assumed linearly proportional to the distance from a fixed origin. However, it is often argued that (Gupta and Gupta, 1977) realistically stretching of the sheet may not necessarily be linear. This situation was beautifully dealt with by Kumaran and Ramanaiah (1996) in their work on a boundary layer flow where, probably for the first time, a general quadratic stretching sheet has been considered. They analyzed their results in terms of a stream function. However, their work was confined to the viscous fluid flow over a permeable stretching sheet. Recently, Khan and Sanjayanand (2004) have extended the work of Kumaran and Ramanaiah (1996) to a viscoelastic boundary layer flow over a general quadratic stretching sheet.

The investigation of heat transfer processes plays an important role in all such theoretical studies. This is due to the fact that a number of metallurgical processes in a polymer processing industry involve the cooling of continuous sheet or filament. The rate of cooling influences a lot the quality of the final product with desired characteristics. Keeping this view we have presented some works on heat transfer in a viscoelastic boundary layer flow over a linearly stretching sheet with a non-uniform prescribed boundary temperature and boundary heat flux (Prasad et al., 2000; 2002). Recently, Ali (1995) investigated the thermal boundary layer by considering a power law stretching surface. A new dimension is added to this investigation by Elbashbeshy (2001) who examined the flow and heat transfer characteristics by considering a exponentially stretching permeable surface. However, the works of Ali (1995) and Elbashbeshy (2001) are confined to the study of a viscous fluid flow only.

In reality, most of the fluids considered in industrial applications are more non-Newtonian in nature, especially of viscoelastic type than viscous type. And also, there may be a situation of a heat source/sink present in the boundary layer. This situation might arise in a flow problem dealing with chemically reactive species (Vajravelu, 1994). Foraboschi and Federico (1990) analysed heat transfer by considering linearly a temperature dependent heat source which is valid for some exothermic processes. Following a similar mathematical formulation for heat source/sink present in the boundary layer we analyse in the present paper the heat transfer processes in a viscoelastic fluid flow over an exponentially stretching sheet. An approximate analytical local similar solution of zero-order is obtained for stream function and velocity distribution by transforming the highly non-linear differential equation into Riccati type and then solving this sequentially. Local similar solutions of temperature for non-isothermal boundary conditions are obtained in the form of confluent hypergeometric functions. The aim of the article is to analyse the effect of several physical parameters like the local viscoelastic parameter, Prandtl number, Reynolds number, Nusselt number, local Eckert number and local source/sink parameters on various momentum and heat transfer characteristics in the presence of a heat source/sink in a boundary layer that develops over an exponentially stretching continuous surface.

\section{Formulation of the problem}

The constitutive equation satisfied by a second order fluid was given by Coleman and Noll (1990), following the postulates of gradually fading memory, as

$$
\boldsymbol{T}=-p \boldsymbol{I}+\mu \boldsymbol{A}_{1}+\alpha_{1} \boldsymbol{A}_{2}+\alpha_{2} \boldsymbol{A}_{\boldsymbol{I}}^{2}
$$

where $T$ is the stress tensor, $p$ is the pressure, $\mu$ is the dynamic viscosity, $\alpha_{1}, \alpha_{2}$ are the material constants with $\alpha_{1}<0$. The stress moduli $\boldsymbol{A}_{\boldsymbol{1}}$ and $\boldsymbol{A}_{2}$ are defined as

$$
\boldsymbol{A}_{\boldsymbol{1}}=(\operatorname{grad} \boldsymbol{q})+(\operatorname{grad} \boldsymbol{q})^{T}
$$




$$
\boldsymbol{A}_{2}=\frac{d \boldsymbol{A}_{\boldsymbol{1}}}{d t}+\boldsymbol{A}_{\boldsymbol{1}}(\operatorname{grad} \boldsymbol{q})+(\operatorname{grad} \boldsymbol{q})^{T} \cdot \boldsymbol{A}_{\boldsymbol{1}}
$$

Although the second order fluid, obeying model Eq.(2.1) with $\alpha_{1}<\alpha_{2}, \alpha_{1}<0$, exhibits some undesirable instability characteristics (Fosdick and Rajagopal, 1979) the second order approximation is valid at low shear rate (Rajagopal et al., 1984).

We consider a steady state laminar flow of an incompressible second order fluid obeying model Eq.(2.1) on a semi-infinite $(y>0)$ stretching sheet. The flow is generated solely due to stretching of the elastic sheet by applying two equal and opposite forces along the $x$-axis keeping the origin fixed (Fig.1). The governing boundary layer equations for such flow situations (Cortell, 1994 and Rajagopal et al., 1987) in the usual notations, are

$$
\begin{aligned}
& \frac{\partial u}{\partial x}+\frac{\partial v}{\partial y}=0 \\
& u \frac{\partial u}{\partial x}+v \frac{\partial u}{\partial y}=\gamma \frac{\partial^{2} u}{\partial y^{2}}-k_{0}\left\{\frac{\partial}{\partial x}\left(u \frac{\partial^{3} u}{\partial y^{2}}\right)+\frac{\partial u}{\partial y} \frac{\partial^{3} v}{\partial y^{3}}+v \frac{\partial^{3} u}{\partial y^{3}}\right\}
\end{aligned}
$$

where $u$ and $v$ are the velocity components in the $x$ and $y$ direction respectively, $\gamma$ is the kinematic coefficient of viscosity and $k_{0}$ is the viscoelasticity. Equation (2.5) has been derived with the assumption that the normal stress is of the same order of magnitude as that of the shear stress, in addition to usual boundary layer approximations.

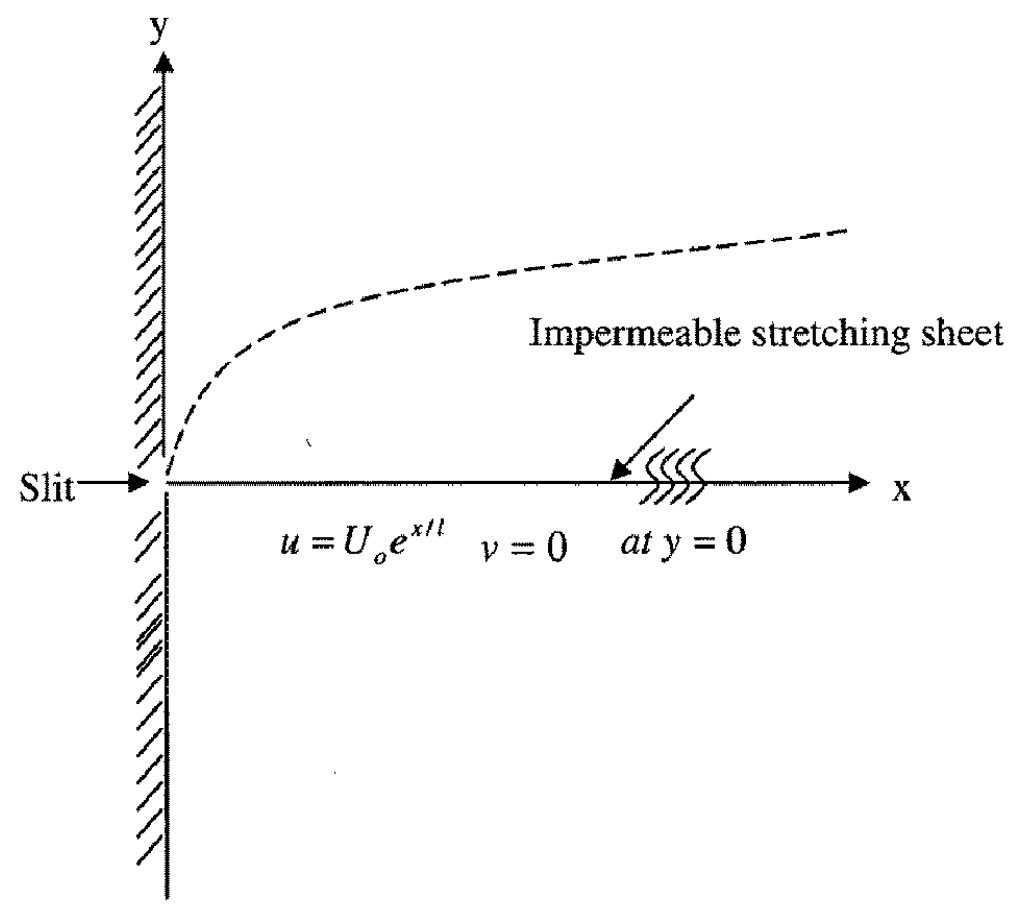

Fig.1. Boundary layer over an impermeable exponential stretching sheet. 
Further, we assume that there may be a temperature dependent heat source/sink present in the boundary layer. In order to determine the temperature distribution in the boundary layer we have to consider a modified energy equation for temperature. There are several mathematical formulations of heat source/sink to take into account the effect of heat generation / absorption. This includes the inclusion of a source/sink term in the energy equation as a constant or as a function of space variable or as a linearly dependent function of temperature. Among all these, a temperature dependent heat source/sink in the form

$$
\frac{Q}{\rho c_{p}}\left(T-T_{\infty}\right)
$$

where $Q$ is constant, is well accepted by the researchers (Vajravelu, 1994; Foraboschi and Federico, 1990; Rollins and Vajravelu, 1991). Hence, following the same type of mathematical formulation and applying the usual boundary layer approximations, the energy equation for temperature may be obtained as the modified energy equation of Mahapatra and Gupta (2004) in the following form.

$$
u \frac{\partial T}{\partial x}+v \frac{\partial T}{\partial y}=\alpha \frac{\partial^{2} T}{\partial y^{2}}+\frac{\mu}{\rho c_{p}}\left(\frac{\partial u}{\partial y}\right)^{2}+\frac{Q}{\rho c_{p}}\left(T-T_{\infty}\right) .
$$

Here $\alpha=\frac{k}{\rho c_{p}}$ is the thermal diffusivity of the fluid, $k$ is the thermal conductivity of the fluid and $\mu$ is the coefficient of viscosity of the fluid. The term $Q$ represents the volumetric rate of heat generation, i.e., heat source when $Q>0$ and heat absorption, i.e., the heat sink when $Q<0$. Other quantities have their usual meanings (Dandapat and Gupta, 1989). In deriving Eq.(2.6) it is assumed that the fluid possesses strong viscous property in comparison with the elastic property. In view of this assumption we neglect the contribution of heat due to elastic deformation. Equation (2.6) is the thermal boundary layer equation which takes into account the viscous dissipation.

\section{Boundary conditions on velocity}

Stretching of the boundary surface is such that the velocity distribution along the axial direction is of exponential order of the axial coordinate. Hence, we employ the following boundary conditions on velocity (Elbashbeshy, 2001).

$$
\begin{aligned}
& u=U_{w}(x)=U_{0} \exp \left(\frac{x}{l}\right), \quad v=0 \quad \text { at } \quad y=0, \\
& u=0, \text { as } \quad y \rightarrow \infty .
\end{aligned}
$$

Here $U_{0}$ is a constant and $l$ is the reference length. Following Elbashbeshy (2001) we have considered the above three boundary conditions for the problem. To the best of the authors' knowledge, all the available literature on boundary layer flows of a viscoelastic fluid over linearly stretching sheets deal with three boundary conditions on velocity, which are one less than the number required to solve the problem uniquely (Rajagopal et al., 1984; 1987; Rollins and Vajravelu 1991; Anderson 1992; Cortell, 1994 and Mahapatra and Gupta, 2004). Troy et al. (1987) gave an exact solution of the problem for linearly stretching boundary conditions. Later, Chang (1989) discussed non-uniqueness of the problem and derived different non-unique solutions. Rajagopal (1987) solved the problem with three boundary conditions only using perturbation expansion. However, Lawrence and Rao (1992) argue that among all the available 
solutions Troy's solution containing exponential terms is physically realistic as it recovers the NavierStokes' solution when the viscoelastic parameter is taken to be zero. In view of this we present, in the next section, physically realistic sequential local similar solutions of the viscoelastic boundary layer equation of the fluid flow over an exponentially stretching sheet.

\section{Solution of the momentum boundary layer equation}

Equation (2.5) may be rewritten in terms of the stream function $\psi(x, y)$, which satisfies the equation of continuity (2.4), by writing

$$
u=\frac{\partial \psi}{\partial y}, \quad v=-\frac{\partial \psi}{\partial x}
$$

Further, the stream function $\psi(x, y)$ may be non-dimensionalised by assuming

$$
\begin{aligned}
& \psi(x, y)=\sqrt{2 \gamma l U_{0}} f(\eta) \exp \left(\frac{x}{2 l}\right) \\
& \eta=y \sqrt{\frac{U_{0}}{2 \gamma l}} \exp \left(\frac{x}{2 l}\right) .
\end{aligned}
$$

Here $f(\eta)$ is the dimensionless stream function and $\eta$ is the pseudo-similarity variable. Making use of Eqs (3.1)-(3.2) in Eq.(2.5) we obtain a fourth order non-linear quasi- ordinary differential equation of the form

$$
2 f_{\eta}^{2}-f f_{\eta \eta}=f_{\eta \eta \eta}-k_{l}^{*}\left[3 f_{\eta} f_{\eta \eta \eta}-\frac{1}{2} f f_{\eta \eta \eta \eta}-\frac{3}{2} f_{\eta \eta}^{2}\right]
$$

Here, $k_{l}^{*}=\frac{k_{0} U_{w}}{\gamma}$ is the dimensionless local viscoelastic parameter.

The boundary conditions on $f$ are

$$
\begin{array}{ll}
f=0, & \text { at } \quad \eta=0, \\
f_{\eta}=0, & \text { as } \eta \rightarrow \infty .
\end{array}
$$

Integrating Eq.(3.4), we obtain

$$
f_{\eta \eta}+f f_{\eta}=-S+\int_{0}^{\eta}\left[3 f_{\eta}^{2}+k_{1}^{*}\left\{3 f_{\eta} f_{\eta \eta \eta}-\frac{1}{2} f f_{\eta \eta \eta \eta}-\frac{3}{2} f_{\eta \eta}^{2}\right\}\right] d \eta .
$$

Applying the boundary condition at $\eta \rightarrow \infty$ in Eq.(3.6) we get 


$$
S=-\int_{0}^{\infty}\left[3 f_{\eta}^{2}+k_{1}^{*}\left\{3 f_{\eta} f_{\eta \eta \eta}-\frac{1}{2} f f_{\eta \eta \eta \eta}-\frac{3}{2} f_{\eta \eta}^{2}\right\}\right] d \eta .
$$

We integrate Eq.(3.6) once again and apply boundary conditions (3.5). This yields

$$
f_{\eta}+\frac{1}{2} f^{2}=1-S \eta+\int_{0}^{\eta}\left[\int_{0}^{\eta_{2}} 3 f_{\eta_{I}}^{2}+k_{1}^{*}\left(3 f_{\eta_{I}} f_{\eta_{I} \eta_{I} \eta_{I}}-\frac{1}{2} f f_{\eta_{I} \eta_{I} \eta_{I} \eta_{I}}-\frac{3}{2} f_{\eta_{I} \eta_{I}}^{2}\right) d \eta_{I}\right] d \eta_{2} .
$$

Now the solution procedure of Eq.(3.8) may be reduced to the sequential solutions of the Riccatitype equations

$$
f_{\eta}^{(n)}+\frac{1}{2} f^{(n)^{2}}=\operatorname{R} . H . S\left(f_{\eta}^{(n-1)}, f_{\eta \eta}^{(n-1)}, f_{\eta \eta \eta}^{(n-1)}, f_{\eta \eta \eta \eta}^{(n-1)}\right) .
$$

This iteration algorithm has to be solved by substituting a suitable zero-order approximation $f_{\eta}^{(0)}(\eta)$ for $f_{\eta}(\eta)$ on the R.H.S.

We assume a zero-order approximation $f_{\eta}^{(0)}(\eta)$ as

$$
f_{\eta}^{(0)}(\eta)=\exp (-S \eta)
$$

which satisfies the boundary conditions at infinity. Integrating Eq.(3.10) and making use of the boundary condition at $\eta=0$ we get

$$
f^{(0)}(\eta)=\frac{1-\exp (-S \eta)}{S}
$$

Substituting all the derivatives of the zero-order approximation $f^{(0)}(\eta)$ into R.H.S of Eq.(3.8) and assuming that first order iteration $f^{(1)}(\eta)$ on the L.H.S of Eq.(3.8) satisfies the boundary conditions at $\eta=0$ of Eq.(3.5) we obtain the value of $S$ as

$$
S=S_{0}=\sqrt{\frac{3}{2\left(1-k_{l}^{*}\right)}} \text { and } f_{\eta \eta}^{(0)}(0)=-S_{0} .
$$

Here, the equation for first-order iteration $f^{(1)}(\eta)$ takes the form

$$
f_{(\eta)}^{(1)}+\frac{1}{2} f^{(1)^{2}}=1+\frac{\left(3+k_{1}^{*} S_{0}^{2}\right)}{4 S_{0}^{2}}\left(e^{-2 S_{0} \eta}-1\right)+\frac{k_{1}^{*}}{2}\left(e^{-S_{0} \eta}-1\right) .
$$


Equation (3.13) is the non-linear Riccati type equation and this can be solved for $f^{(1)}(\eta)$. However, we shall make use of zero-order solutions $f^{(0)}(\eta)$ and $f_{\eta}^{(0)}(\eta)$ given by Eqs (3.11) and (3.10) respectively, as these solutions match very close the numerical solution of Eq.(3.4) (Fig.2). Further, the use of the zeroorder solutions $f^{(0)}(\eta)$ and $f_{\eta}^{(0)}(\eta)$ of the form of Eqs (3.11) and (3.10), respectively enables us to obtain the solution of the heat transfer Eq.(2.6) in the form of confluent hypergeometric functions.

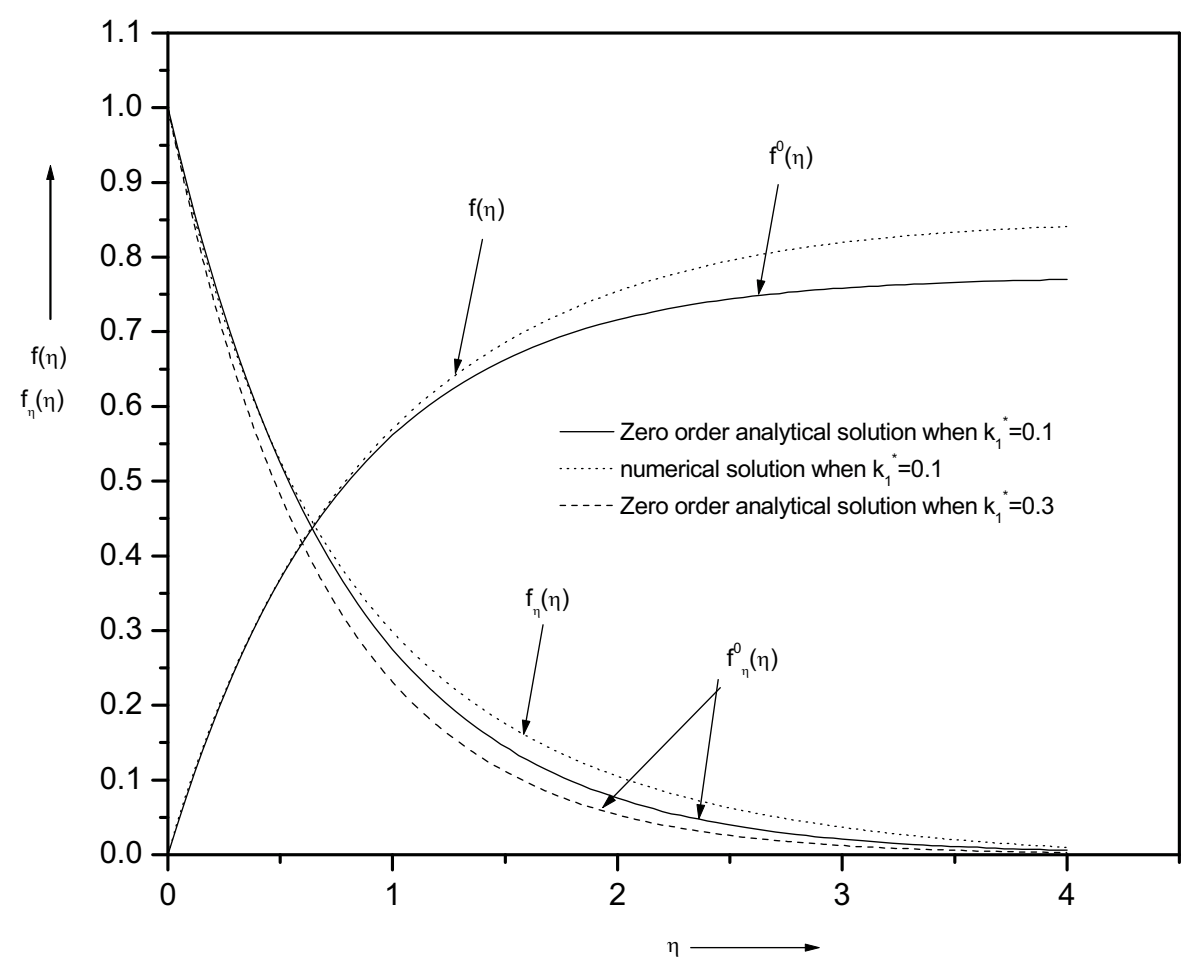

Fig.2. Profiles for $f(\eta)$ and $f_{\eta}(\eta)$ obtained from numerical as well as zero order analytical solution when $k_{1}=0.1$ and $k_{1}=0.3$.

The dimensionless local skin friction coefficient $C_{f}$ is expressed as

$$
\begin{aligned}
C_{f} & =-\frac{\left(\gamma \frac{\partial u}{\partial y}-k_{0}\left\{u \frac{\partial^{2} u}{\partial x \partial y}-2 \frac{\partial u}{\partial y} \frac{\partial v}{\partial y}\right\}\right)}{U_{0}^{2} \exp \left(\frac{2 x}{l}\right)} \text { at } y=0 \\
& =\frac{S_{0}}{\sqrt{2 \operatorname{Re}}}\left[1-\frac{7}{2} k_{l}^{*}\right]
\end{aligned}
$$

Here, $\operatorname{Re}=\frac{U_{w} l}{\gamma}$ is the non-dimensional Reynolds number. 


\section{Solution of the heat transfer equation}

We consider two general cases of non-isothermal temperature boundary conditions, namely:

(A) boundary with prescribed exponential order surface temperature (PEST); and

(B) boundary with prescribed exponential order heat flux (PEHF)

in order to solve the temperature Eq.(2.6)

\section{Case A: Prescribed Exponential Order Surface Temperature (PEST)}

We employ the following non-isothermal boundary condition on temperature in the PEST case

$$
\begin{aligned}
& T=T_{w}=T_{\infty}+T_{0} \exp \left(\frac{v_{0} x}{2 l}\right) \quad \text { at } \quad y=0, \\
& T=T_{\infty} \text { as } \quad y \rightarrow \infty
\end{aligned}
$$

where $v_{0}$ and $T_{0}$ are the parameters of temperature distribution on the stretching surface. $T_{\infty}$ is the temperature of the ambient fluid far away from the stretching surface.

Now we define a dimensionless temperature variable $\theta(\eta)$ in order to obtain a local similarity solution of temperature Eq.(2.6) as follows

$$
\theta(\eta)=\frac{T-T_{\infty}}{T_{w}-T_{\infty}}
$$

where $T_{w}-T_{\infty}$ is given by Eq.(4.1). With this dimensionless variable the temperature Eq.(2.6) takes the form

$$
\theta_{\eta \eta}+\operatorname{Pr} f \theta_{\eta}-\operatorname{Pr}\left(v_{0} f_{\eta}-\beta^{*}\right) \theta=-\operatorname{Pr} \mathrm{E} f_{\eta \eta}^{2}
$$

where $\operatorname{Pr}=\frac{\gamma}{\alpha}$ is the Prandtl number, $\mathrm{E}=\frac{U_{0}^{2}}{c_{p} T_{0}}\left(\frac{U_{w}}{U_{0}}\right)^{\frac{4-v_{0}}{2}}$ is the local Eckert number and $\beta^{*}=\beta \frac{U_{0}}{U_{w}}$ is the non-dimensional local source/sink parameter. Here $\beta^{*}>0$ represents source and $\beta^{*}<0$ represents sink.

Boundary conditions of temperature in a non-dimensional form are

$$
\begin{aligned}
& \theta(0)=1, \\
& \theta(\infty)=0 .
\end{aligned}
$$

We proceed to solve Eq.(4.2) by using the zero-order approximation of $f(\eta)$ given by Eq.(3.11). Further, we introduce a new dimensionless variable

$$
\xi=-\frac{\operatorname{Pr}}{S_{0}^{2}} e^{-S_{0} \eta}
$$


Substitution of Eqs (4.4) and (3.11) in Eqs (4.2) - (4.3) leads to the following two-point boundary value problem.

$$
\xi \theta_{\eta \eta}+\left(1-\operatorname{Pr}^{*}-\xi\right) \theta_{\xi}+\left(v_{0}+\frac{\operatorname{Pr}^{*} \beta^{*}}{\xi}\right) \theta=\frac{-E S_{0}^{2}}{\operatorname{Pr}^{*}} \xi
$$

subject to the boundary conditions

$$
\begin{aligned}
& \theta\left(\xi=-\operatorname{Pr}^{*}\right)=1, \\
& \theta(\xi=0)=0
\end{aligned}
$$

where $\operatorname{Pr}^{*}=\frac{\operatorname{Pr}}{S_{0}^{2}}$ is the modified Prandtl number.

We assume the solution of Eq.(4.5) in the form

$$
\theta(\xi)=\theta_{c}(\xi)+\theta_{p}(\xi)
$$

$\theta_{c}(\xi)$ is the complementary solution and $\theta_{p}(\xi)$ stands for particular integral. Using the boundary conditions of Eq.(4.6) we obtain a complementary solution of Eq.(4.5) in the following form of a confluent hypergeometric function

$$
\theta_{c}(\xi)=A \xi^{\frac{a_{0}+b_{0}}{2}} M\left(\frac{a_{0}+b_{0}}{2}-v_{0}, a_{0}+b_{0}+1-\operatorname{Pr}^{*}, \xi\right)
$$

where

$$
a_{0}=\operatorname{Pr}^{*}, b_{0}=\sqrt{\operatorname{Pr}^{* 2}-4 \beta^{*}} \text { and } A \text { is the arbitrary constant. }
$$

The function $M\left(a_{0}, b_{0}, z\right)$ is Kummer's function and it is defined (Abramowitz and Stegun, (1972) by

$$
\begin{aligned}
& M\left(a_{0}, b_{0}, z\right)=1+\sum_{n=1}^{\infty} \frac{\left(a_{0}\right)_{n} z^{n}}{\left(b_{0}\right)_{n} n !} \\
& \left(a_{0}\right)_{n}=a_{0}\left(a_{0}+1\right)\left(a_{0}+2\right)------\left(a_{0}+n-1\right), \\
& \left(b_{0}\right)_{n}=b_{0}\left(b_{0}+1\right)\left(b_{0}+2\right)-------\left(b_{0}+n-1\right) .
\end{aligned}
$$

It is to be noted that Eq.(4.5) admits a closed form particular solution if only we choose $v_{0}=2$ and this is obtained as 


$$
\theta_{p}(\xi)=\frac{-\mathrm{E} S_{0}^{2}}{\operatorname{Pr}^{*}\left(4-2 \operatorname{Pr}^{*}+\beta^{*} \operatorname{Pr}^{*}\right)} \xi^{2}
$$

Substituting Eqs (4.9) and (4.10) in Eq.(4.8) we get the solution of Eq.(4.5) as

$$
\theta(\xi)=A \xi^{\frac{a_{0}+b_{0}}{2}} M\left(\frac{a_{0}+b_{0}}{2}-2, a_{0}+b_{0}+1-\operatorname{Pr}^{*}, \xi\right)-\frac{E S_{0}^{2}}{\operatorname{Pr}^{*}\left(4-2 \operatorname{Pr}^{*}+\beta^{*} \operatorname{Pr}^{*}\right)} \xi^{2},
$$

which satisfies the second boundary condition at $\xi=0$ of Eq.(4.6). To determine the arbitrary constant $A$ we use the other boundary condition of Eq.(4.6) and obtain

$$
A=\frac{1+\frac{\mathrm{E} S_{0}^{2} \operatorname{Pr}^{*}}{\left(-\operatorname{Pr}^{*}\right)^{\frac{a_{0}+b_{0}}{2}} M\left(\frac{a_{0}+b_{0}}{2}-2, a_{0}+b_{0}+1-\operatorname{Pr}^{*},-\operatorname{Pr}^{*}\right)}}{.}
$$

Therefore, after substituting the value of $A$, we rewrite the solution in terms of the variable $\eta$ and we get

$$
\theta(\eta)=\frac{\left(1-C_{1}\right) e^{-S_{0}\left(\frac{a_{0}+b_{0}}{2}\right) \eta} M\left(\frac{a_{0}+b_{0}}{2}-2, a_{0}+b_{0}+1-\operatorname{Pr}^{*},-\operatorname{Pr}^{*} e^{-S_{0} \eta}\right)}{M\left(\frac{a_{0}+b_{0}}{2}-2, a_{0}+b_{0}+1-\operatorname{Pr}^{*},-\operatorname{Pr}^{*}\right)}+C_{1} e^{-2 S_{0} \eta}
$$

where

$$
C_{1}=\frac{-\mathrm{E} S_{0}^{2} \operatorname{Pr}^{*}}{\left(4-2 \operatorname{Pr}^{*}+\beta^{*} \operatorname{Pr}^{*}\right)}
$$

We know that the wall temperature gradient is an important parameter associated with the heat transfer analysis. Hence, we obtain an expression for the dimensionless wall temperature gradient $\theta_{\eta}(0)$ as

$$
\theta_{\eta}(0)=\left(1-C_{1}\right)\left[\left(\frac{a_{0}+b_{0}-4}{2\left(a_{0}+b_{0}+1-\operatorname{Pr}^{*}\right)}\right) S_{0} \operatorname{Pr}^{*} \frac{M\left(\frac{a_{0}+b_{0}}{2}-1, a_{0}+b_{0}+2-\operatorname{Pr}^{*},-\operatorname{Pr}^{*}\right)}{M\left(\frac{a_{0}+b_{0}}{2}-2, a_{0}+b_{0}+1-\operatorname{Pr}^{*},-\operatorname{Pr}^{*}\right)}-S_{0}\left(\frac{a_{0}+b_{0}}{2}\right)\right]-2 C_{1} S_{0} .
$$

Substituting the values of $k_{l}^{*}=0, \quad \beta^{*}=0$ in the Eqs (4.13) - (4.15) we obtain the result of Elbashbeshy (2001) in the absence of suction. Conventionally, heat transfer analysis is carried out by means of dimensionless number of temperature gradient, known as the local Nusselt number. The local Nusselt number in the present case is derived as 


$$
\begin{aligned}
\mathrm{Nu} & =\frac{x}{\left(T_{w}-T_{\infty}\right)}\left(\frac{\partial T}{\partial y}\right)_{y=0} \\
& =\theta_{\eta}(0) \frac{x}{l \sqrt{2}} \sqrt{\operatorname{Re}}
\end{aligned}
$$

where $\mathrm{Re}$ is the local Reynolds number and it is defined as

$$
\operatorname{Re}=\frac{U_{w} l}{\gamma} .
$$

\section{Case B: Prescribed Exponential Order Heat Flux (PEHF)}

In this case we assume that the stretching surface is subjected to exponential order heat flux with the axial direction. Therefore we employ the following prescribed exponential law heat flux boundary condition.

$$
\begin{aligned}
& -k\left(\frac{\partial T}{\partial y}\right)_{w}=T_{1} \exp \left(\frac{v_{1}+1}{2 l}\right) x \quad \text { at } \quad y=0, \\
& T \rightarrow T_{\infty} \quad \text { as } \quad y \rightarrow \infty .
\end{aligned}
$$

Here, $v_{1}$ and $T_{1}$ are the parameters of temperature distribution on the stretching surface.

We seek a local similar solution for temperature and so we define the dimensionless temperature variable as follows

$$
g(\eta)=\frac{T-T_{\infty}}{\frac{T_{1}}{k} \sqrt{\frac{2 v_{1} l}{U_{0}}} \exp \left(\frac{v_{1} x}{2 l}\right)}
$$

Using this dimensionless variable and Eqs (3.1) - (3.3) in Eq.(2.6) we obtain the dimensionless temperature equation as

$$
g_{\eta \eta}+\operatorname{Pr} f g_{\eta}-\operatorname{Pr}\left(v_{1} f_{\eta}-\beta^{*}\right) g=-\operatorname{Pr} \mathrm{E} f_{\eta \eta}^{2}
$$

where

$$
\mathrm{E}=\frac{U_{0}^{2} k}{c_{p} T_{1} \sqrt{\frac{2 v_{l} l}{U_{0}}}}\left(\frac{U_{w}}{U_{0}}\right)^{\frac{4-v_{l}}{2}} \quad \text { and } \quad \operatorname{Pr}=\frac{\gamma}{\alpha}
$$

are the local Eckert number and the Prandtl number, respectively.

The non-dimensional temperature boundary conditions are 


$$
\begin{aligned}
& g_{\eta}(0)=-1, \\
& g(\infty)=0 .
\end{aligned}
$$

Equation (4.19) is of the same form as Eq.(4.2). However, the first boundary condition of Eq.(4.3) differs from that of the boundary condition (4.21). Therefore following the same procedure as described in the PEST case and making use of the boundary conditions of Eq.(4.21) we derive the solution for $g(\eta)$ in the following form of a confluent hypergeometric function.

$$
g(\eta)=C_{2} e^{-S_{0} \frac{a_{0}+b_{0}}{2} \eta} M\left[\frac{a_{0}+b_{0}}{2}-2, a_{0}+b_{0}+1-\operatorname{Pr}^{*},-\operatorname{Pr}^{*} e^{-s_{0} \eta}\right]+C_{1} e^{-2 s_{0} \eta}
$$

The expression for the constant $C_{2}$ is

$$
C_{2}=\frac{\left\{1+\frac{2 \mathrm{E} S_{0}^{3} \operatorname{Pr}^{*}}{\left(4-2 \mathrm{Pr}^{*}+\beta^{*}\right) \operatorname{Pr}^{*}}\right\}}{\left\{\begin{array}{l}
S_{0}\left(\frac{a_{0}+b_{0}}{2}\right) M\left(\frac{a_{0}+b_{0}}{2}-2, a_{0}+b_{0}+1-\operatorname{Pr}^{*},-\operatorname{Pr}^{*}\right)- \\
\frac{\left(a_{0}+b_{0}-4\right)}{2\left(a_{0}+b_{0}+1-\operatorname{Pr}^{*}\right)} \operatorname{Pr}^{*} S_{0} M\left(\frac{a_{0}+b_{0}}{2}-1, a_{0}+b_{0}+2-\operatorname{Pr}^{*},-\operatorname{Pr}^{*}\right)
\end{array}\right\}},
$$

and the expression for $C_{1}$ is given by Eq.(4.14) and expressions for $a_{0}$ and $b_{0}$ are given by Eq.(4.9).

In this boundary heating process (PEHF) the dimensionless wall temperature $g(0)$ is obtained as

$$
g(0)=C_{2} M\left[\frac{a_{0}+b_{0}}{2}-2, a_{0}+b_{0}+1-\operatorname{Pr}^{*},-\operatorname{Pr}^{*}\right]+C_{1}
$$

The expression for the dimensional local wall temperature is

$$
T_{w}=T_{\infty}+\frac{T_{1}}{k} \sqrt{\frac{2 v_{l} l}{U_{0}}} \exp \left(\frac{v_{l} x}{2 l}\right) g(0) .
$$

\section{Results and discussion}

A comprehensive mathematical model on momentum and heat transfer phenomena has been formulated for a boundary layer viscoelastic fluid flow over an exponentially stretching impermeable sheet in the presence of local heat source/sink. In the process of deriving the mathematical solution, the highly non-linear partial differential equations characterizing flow and heat transfer phenomena have been converted into a set of non-linear quasi-ordinary differential equations by applying suitable pseudo--similar transformations. Sequential solutions of the transformed non-dimensional stream function equation are obtained by solving the non-linear Riccati type equation analytically. The zero-order approximate solution for the dimensionless stream function $f(\eta)$ which satisfies all the boundary conditions has been obtained analytically. First-order approximate solution of $f(\eta)$ can also be derived analytically from Eq.(3.13) in the 
form of a confluent hypergeometric Whittaker's function (Khan). However, numerical solutions of Eq.(3.4) for $f(\eta)$ and $f_{\eta}(\eta)$ may be obtained. In order to solve the forth order non-linear Eq.(3.4) numerically it is essential to have one more boundary condition in addition to the given three boundary conditions of Eq.(3.5). We generate the fourth boundary condition by substituting the boundary conditions of Eq.(3.5) in Eq.(3.4) and we get

$$
f_{\eta \eta \eta}(0)=\frac{4-3 k_{1}^{*} f_{\eta \eta}^{2}(0)}{2\left(1-3 k_{l}^{*}\right)} .
$$

Now using all the four boundary conditions, we obtain a numerical solution of the differential Eq.(3.4) for $f(\eta)$ and $f_{\eta}(\eta)$ by employing the Runge-Kutta fourth order method involving shooting. An analysis of Fig.2. reveals that numerical solutions match very well the solutions of zero-order $f^{(0)}(\eta)$ and $f_{\eta}^{(0)}(\eta)$ in the region very close to the boundary. In view of this we have considered a zero-order approximate solution of $f(\eta)$ and obtained the exact analytical solution of the heat transfer equation in the form of confluent hypergeometric functions (Eq.(4.13) - (4.15) and (4.22) - (4.23)). All these solutions involve an exponential dependence of (i) the pseudo-similarity variable $\eta$ (ii) stream function $f(\eta)$ (iii) velocity component $f_{\eta}(\eta)$ and (iii) temperature distribution $\theta(\eta)$ and $g(\eta)$ on the coordinate $\mathrm{x}$ along the direction of stretching.

Some qualitative behaviors of the flow and heat transfer characteristics, are demonstrated in Figs 2-6 and Tabs 1-2. The graphs for the non-dimensional velocity profile $f_{\eta}(\eta)$ for different values of the local viscoelastic parameter $k_{l}^{*}$ are also plotted in Fig.2. From this figure we notice that the effect of the local viscoelastic parameter $k_{l}^{*}$ decreases velocity throughout the boundary layer flow field which is quite obvious. The graphs of the non-dimensional local skin-friction parameter $C_{f}$ vs. the local viscoelastic parameter $k_{l}^{*}$ for different values of the local Reynolds number Re are shown in Fig.3. This figure demonstrates that the increase of the non-dimensional local viscoelastic parameter $k_{1}^{*}$ leads to the decrease of the local skin-friction parameter $C_{f}$. There will be a separation of the boundary layer for the value of the local viscoelastic parameter $k_{l}^{*}=\frac{2}{7}$. This result is the consequence of the fact that an elastic property in a viscoelastic fluid reduces frictional force. This result has significant bearing on the polymer processing industry, as the choice of a higher order viscoelastic fluid would reduce the power consumption for stretching the boundary sheet. The effect of the local Reynolds number on the skin-friction coefficient is also seen to reduce the local skin-friction coefficient $C_{f}$ as a reduction of the viscous property of the fluid results in the decrease of frictional force. Figures $4 \mathrm{a}$ and $4 \mathrm{~b}$ demonstrate the effect of the Prandtl number Pr and local Eckert number E on the non-dimensional temperature distribution when the heat source parameter of strength $\beta^{*}=0.3$ is present in the boundary layer for PEST and PEHF cases, respectively. These graphs show that the increase of the Prandtl number Pr results in the decrease of temperature distribution at a particular point in the flow region. This behaviour occurs because there would be a decrease of the thermal boundary layer thickness with the increase of values of the Prandtl number Pr as a result of a slow rate of thermal diffusion. However, in the presence of viscous dissipation $(\mathrm{E} \neq 0)$ there may be a higher temperature distribution near the boundary for higher values of the Prandtl number. It is interesting to note that this region of higher temperature distribution for higher values of the Prandtl number Pr decreases with the increase of the viscoelastic parameter $k_{l}^{*}$. The wall temperature 
distribution is seen to be at unity in the PEST case for all values of $\operatorname{Pr}, \mathrm{E}$ and $k_{1}^{*}$. However, it may be other than unity in the PEHF case due to the adiabatic temperature boundary condition. Increasing the values of the local viscoelastic parameter $k_{l}^{*}$ is seen to increase the temperatures distribution in the boundary layer, which is the consequence of the property of non-Newtonian viscoelastic fluid that leads to the increases of thermal boundary layer thickness. The results of the PEHF case are qualitatively similar to those of PEST but quantitatively they differ. It is of interest to note that the effect of increasing the values of the local Eckert number $E$ increases temperature distribution in the flow region for both the cases of PEST and PEHF. This is because the heat energy generated in the fluid due to frictional heating results in the temperature enhancement. In conformity with reality we notice that temperature distribution attains its peak value near the boundary in the presence of viscous dissipation.

Table 1. Wall temperature gradient $-\theta_{\eta}(0)$ in PEST case for different values of Prandtl number Pr, Eckert number E, viscoelastic parameter $k_{1}^{*}$, and heat Sourc/Sink parameter $\beta^{*}$.

\begin{tabular}{|l|l|l|l|l|l|}
\hline \multirow{2}{*}{$k_{1}$} & \multirow{2}{*}{$\operatorname{Pr}$} & \multirow{2}{*}{ E } & \multicolumn{2}{|l|}{$-\theta_{\eta}(0)$} & \multicolumn{2}{|l|}{} \\
\cline { 4 - 6 } & & & with $\beta^{*}=0.2$ & with $\beta^{*}=0.0$ & with $\beta^{*}=-0.2$ \\
\hline $10^{-9}$ & 5 & 0 & 3.21 & $3.26(3.257)$ & 3.31 \\
0.2 & & & 3.15 & $3.22(3.219)$ & 3.28 \\
\hline $10^{-9}$ & 8 & & 4.17 & 4.20 & 4.24 \\
0.2 & & & 4.12 & 4.17 & 4.21 \\
\hline $10^{-9}$ & 5 & 0.5 & 2.26 & 2.50 & 2.66 \\
0.2 & & & 1.86 & 2.32 & 2.55 \\
\hline $10^{-9}$ & 8 & & 2.94 & 3.15 & 3.31 \\
0.2 & & & 2.62 & 2.91 & 3.12 \\
\hline
\end{tabular}

(The values in the parenthesis are results of Khan and Sanjayanand (2005) which match our results)

Table 2. Wall temperature $g(0)$ in PEHF case for different values of Prandtl number Pr, Eckert number E, viscoelastic parameter $k_{l}^{*}$ and heat Sourc/Sink parameter $\beta^{*}$.

\begin{tabular}{|l|l|l|l|l|l|}
\hline \multirow{2}{*}{$k_{1}$} & \multirow{2}{*}{ Pr } & \multirow{2}{*}{ E } & \multicolumn{2}{l|}{$g(0)$} & \multicolumn{2}{l|}{} \\
\cline { 4 - 6 } & & & with $\beta^{*}=0.2$ & with $\beta^{*}=0.0$ & with $\beta^{*}=-0.2$ \\
\hline $10^{-9}$ & 5 & 0 & 0.311 & $0.307(0.307)$ & 0.302 \\
0.2 & & & 0.317 & $0.311(0.311)$ & 0.305 \\
\hline $10^{-9}$ & 8 & & 0.243 & 0.238 & 0.236 \\
0.2 & & & 0.240 & 0.239 & 0.237 \\
\hline $10^{-9}$ & 5 & 0.5 & 0.607 & 0.539 & 0.497 \\
0.2 & & & 0.729 & 0.591 & 0.527 \\
\hline $10^{-9}$ & 8 & & 0.534 & 0.488 & 0.454 \\
0.2 & & & 0.607 & 0.542 & 0.497 \\
\hline
\end{tabular}

(The values in the parenthesis are results of Khan and Sanjayanand (2005) which match the results obtained in the present study) 


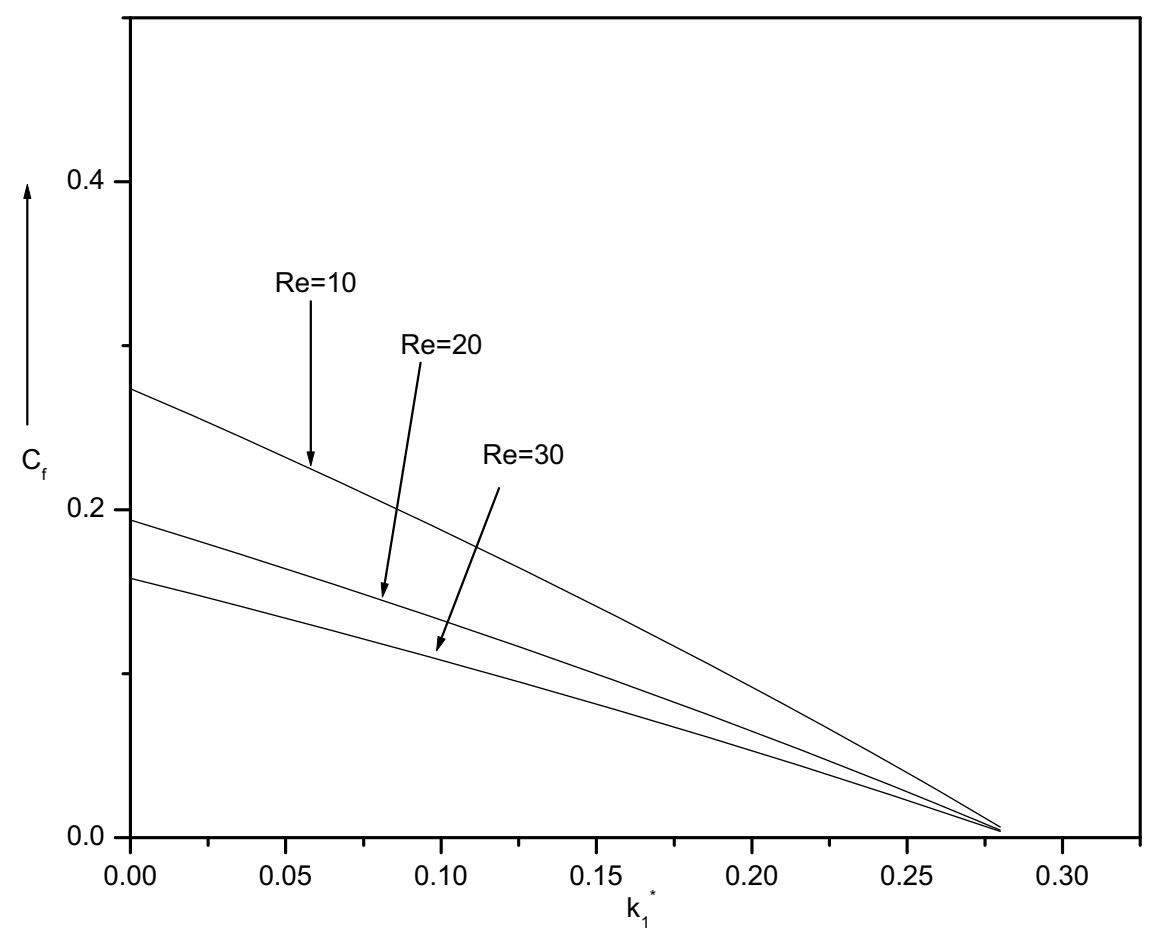

Fig.3. Graph of skinfriction parameter $C_{f} v s$ viscoelastic parameter $k_{l}^{*}$ for different values of Reynolds number Re.

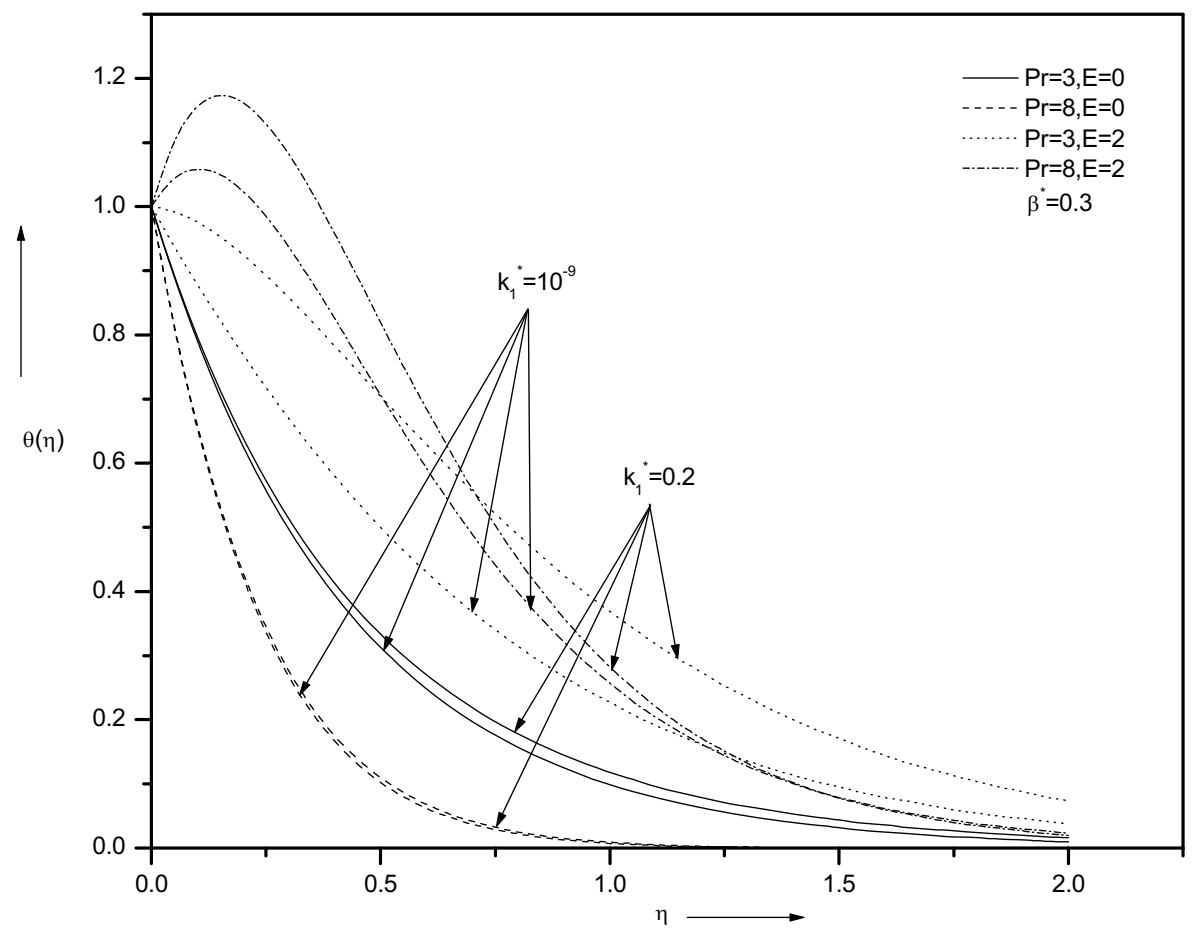

Fig.4a. Dimensionless temperature profile $\theta(\eta)$ for various values of Prandtl number Pr and Eckert number $\mathrm{E}$ in PST case when $\beta^{*}=0.3$. 


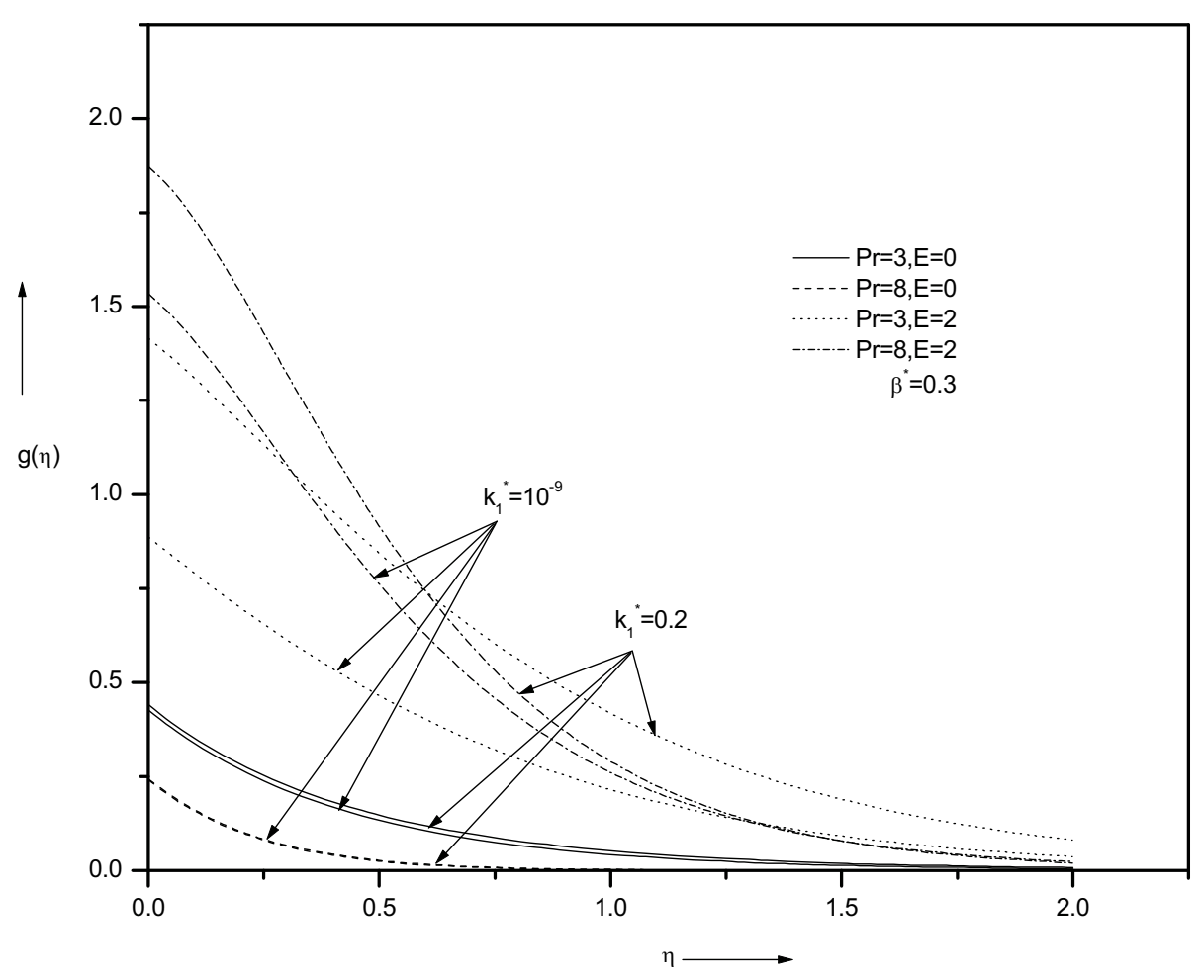

Fig.4b. Dimensionless temperature profile $g(\eta)$ for various values of Prandtl number Pr and Eckert number E in PEHF case when $\beta^{*}=0.3$.

The dimensionless temperature profile $\theta(\eta)$ for various values of the Prandtl number Pr and local Eckert number E are shown in Fig.5a. and Fig.5b. for PEST and PEHF cases, respectively, when there is no local heat source/sink present in the boundary layer. A comparison of Fig.4a and Fig.5a shows that the region of higher temperature near the boundary for higher values of the Prandtl number reduces significantly in the absences of heat source/sink in the boundary layer. Other qualitative features of the graphs of Fig.5a and Fig.5b are exactly the same as those in Figs $4 \mathrm{a}$ and $4 \mathrm{~b}$, respectively, except for a reduction of magnitude of temperature throughout the boundary layer. The profile for dimensionless temperature $\theta(\eta)$ for the same data, as those in Fig.5, is shown in Fig.6 when there is local heat sink present in the boundary layer. From this figure we observe that temperature attains its peak value near the boundary for lower values of the Prandtl number and higher values of the local viscoelastic parameter in the presence of local heat sink and viscous heat sink dissipation in the boundary layer. A comparative study of Fig.5. and Fig.6 shows that the effect of heat sink present in the region causes a reduction of the values of temperature throughout the boundary layer.

We show the numerical values of the non-dimensional wall temperature gradient $-\theta_{\eta}(0)$ in the PEST case for different values of the Prandtl number Pr, local Eckert number E and local viscoelastic parameter $k_{1}^{*}$ in Tab.1. An analysis of the tabulated values reveals that the increase of the values of the Prandtl number Pr results in the increase of the values of the wall temperature gradient $-\theta_{\eta}(0)$. By increasing the values of the local viscoelastic parameter $k_{l}^{*}$ we notice that the wall temperature gradient $-\theta_{\eta}(0)$ is decreased. The effect of viscous dissipation $(E \neq 0)$ reduces the wall temperature gradient 
$-\theta_{\eta}(0)$. The effect of the local source and sink parameter decreases and increases the temperature gradient $-\theta_{\eta}(0)$, respectively. The wall temperature gradient $-\theta_{\eta}(0)$ attains a minimum value with the decrease of the Prandtl number Pr and increase of the local viscoelastic parameter $k_{l}^{*}$ and local Eckert number E in the presence of heat/source. The rate of heat transfer can be controlled by suitably choosing the values of the Prandtl number, local Eckert number, local viscoelastic parameter and local source/sink parameter.

Table 2 is plotted for the different values of the Prandtl number Pr, local Eckert number E and local viscoelastic parameter $k_{l}^{*}$ for the non-dimensional wall temperature $g(0)$ in the PEHF case. An analysis of the tabular results shows that as the value of the Prandtl number Pr increases the non-dimensional wall temperature $g(0)$ decreases and increasing the values of the local viscoelastic parameter $k_{l}^{*}$ leads to the increase of the non dimensional wall temperature $g(0)$. The effect of the local source and sink parameter $\beta^{*}$ is to increase and decrease in surface temperature respectively. Hence we can control the surface temperature distribution by changing the values of the Prandtl number, local Eckert number, local viscoelastic parameter and local source/sink parameter.

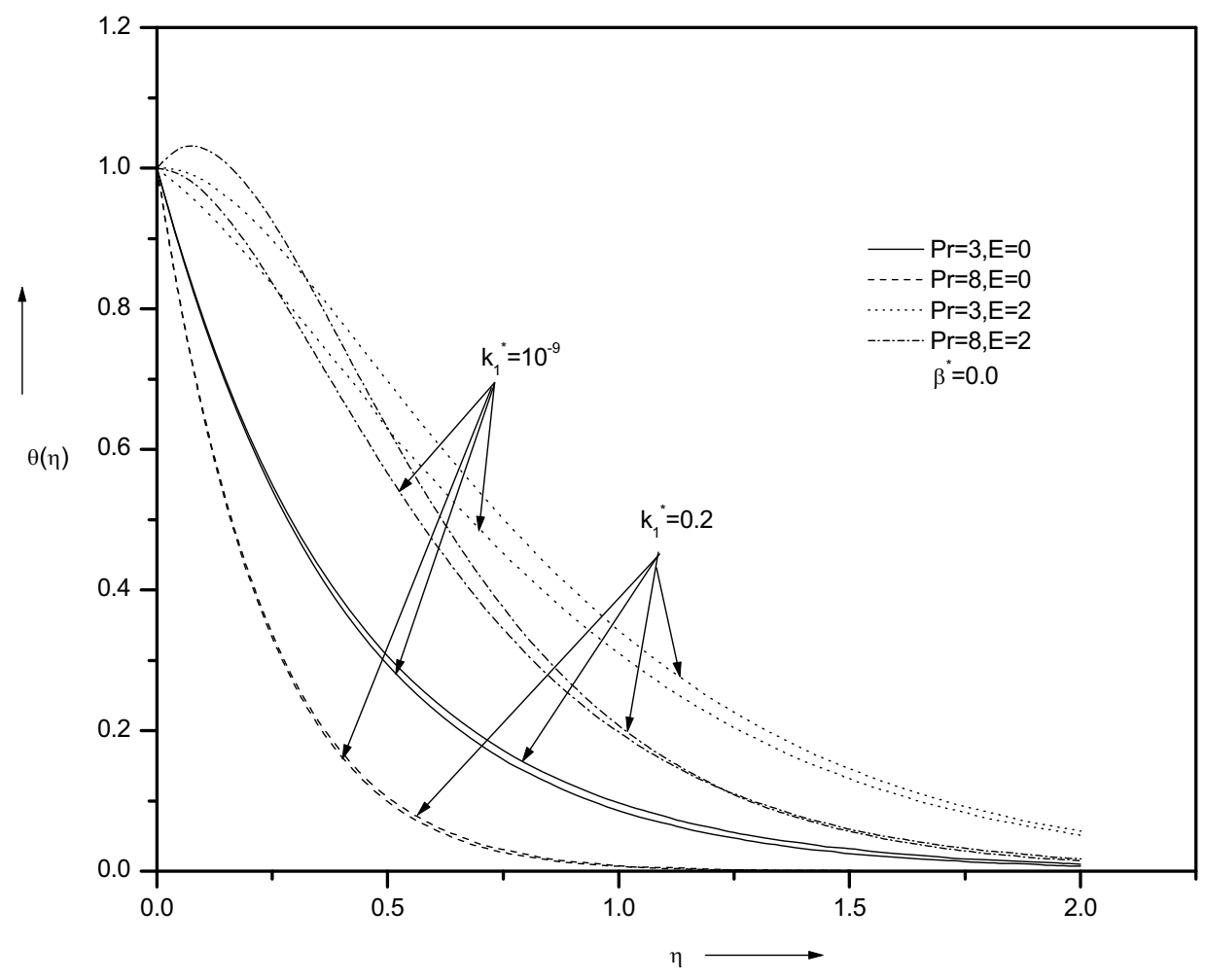

Fig.5a. Dimensionless temperature profile $\theta(\eta)$ for various values of Prandtl number Pr and Eckert number E in PEST case when $\beta^{*}=0.0$. 


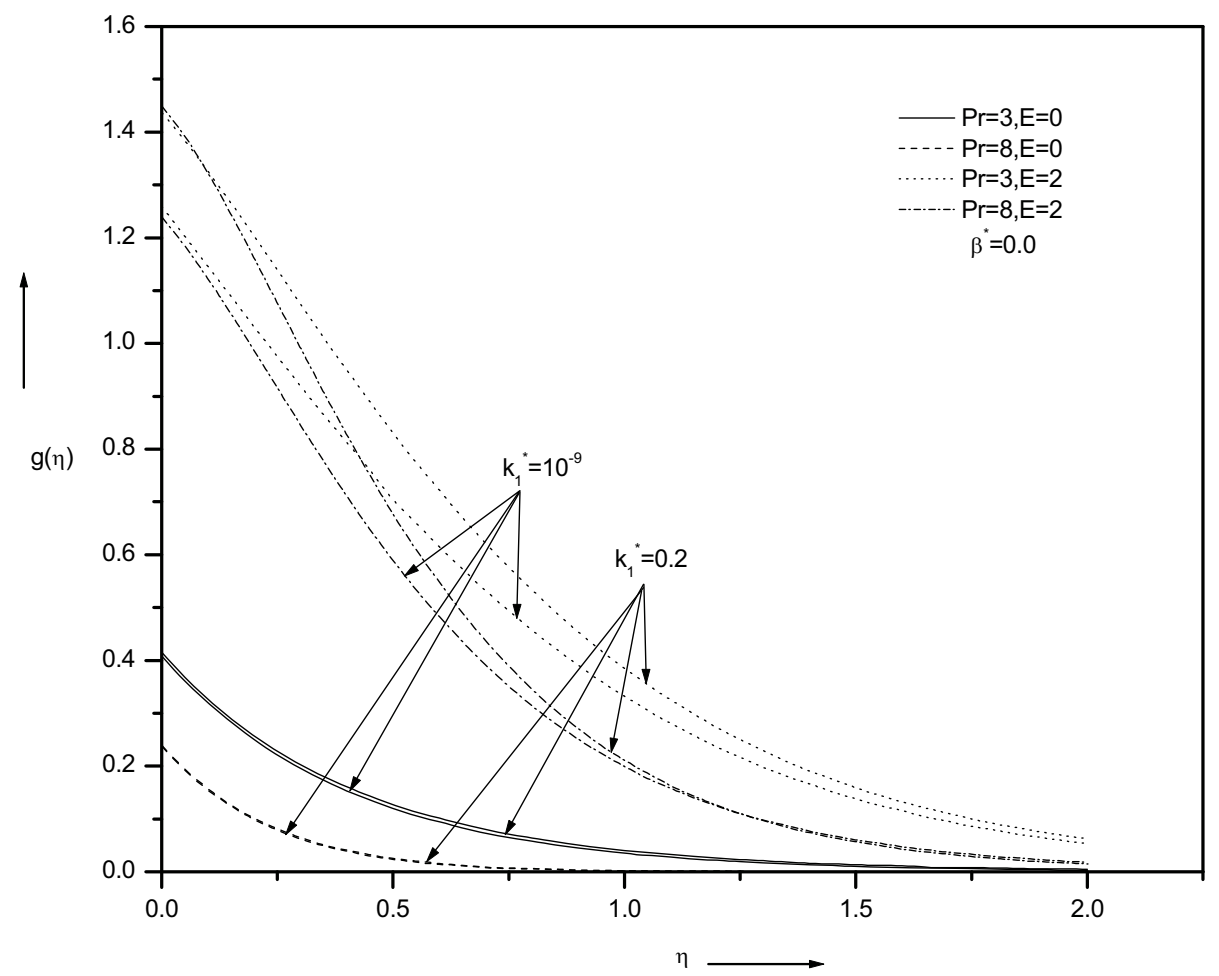

Fig.5b. Dimensionless temperature profile $g(\eta)$ for various values of Prandtl number Pr and Eckert number $\mathrm{E}$ in PEHF case when $\beta^{*}=0.0$.

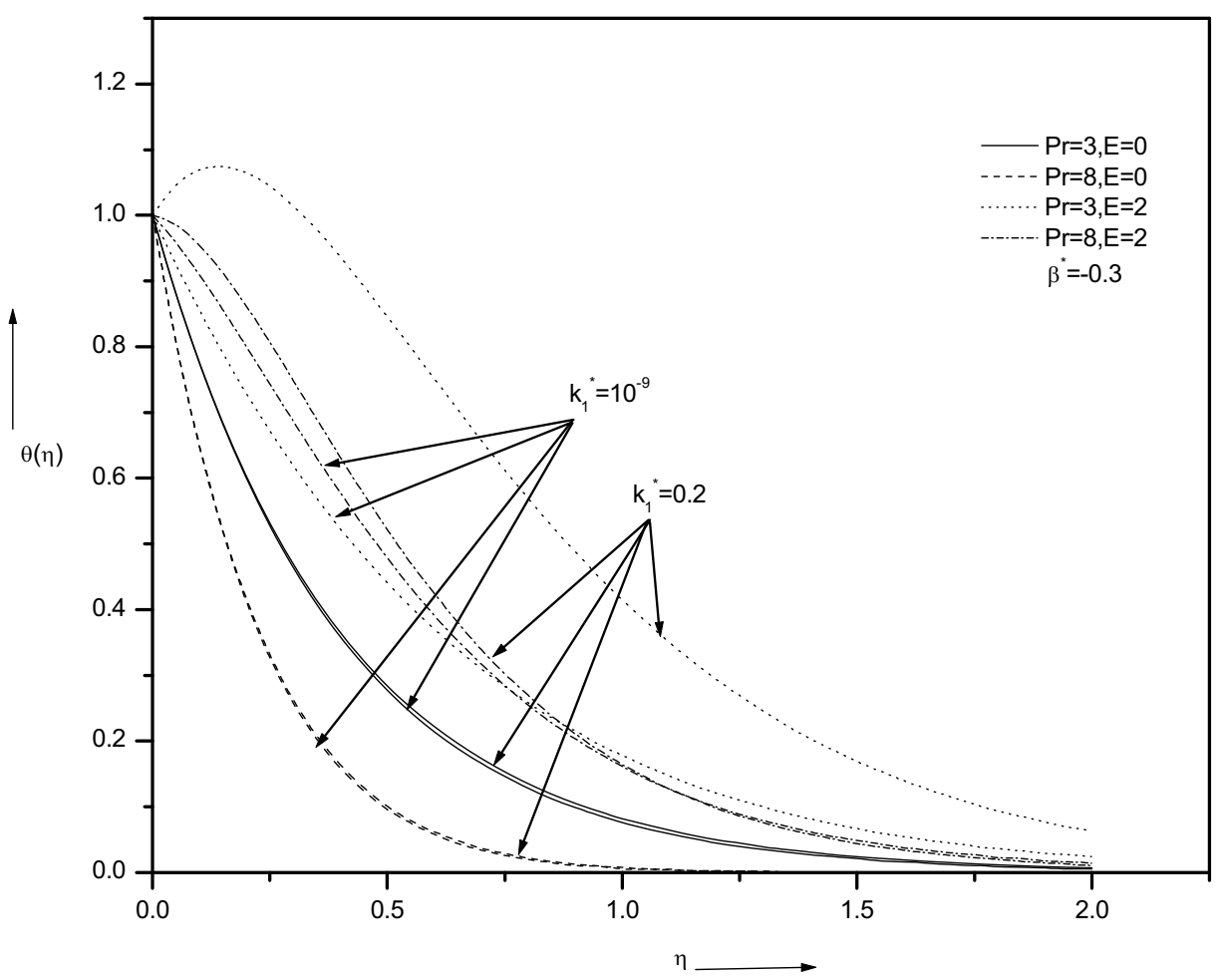

Fig.6a. Dimensionless temperature profile $\theta(\eta)$ for various values of Prandtl number Pr and Eckert number E in PEST case when $\beta^{*}=-0.3$. 


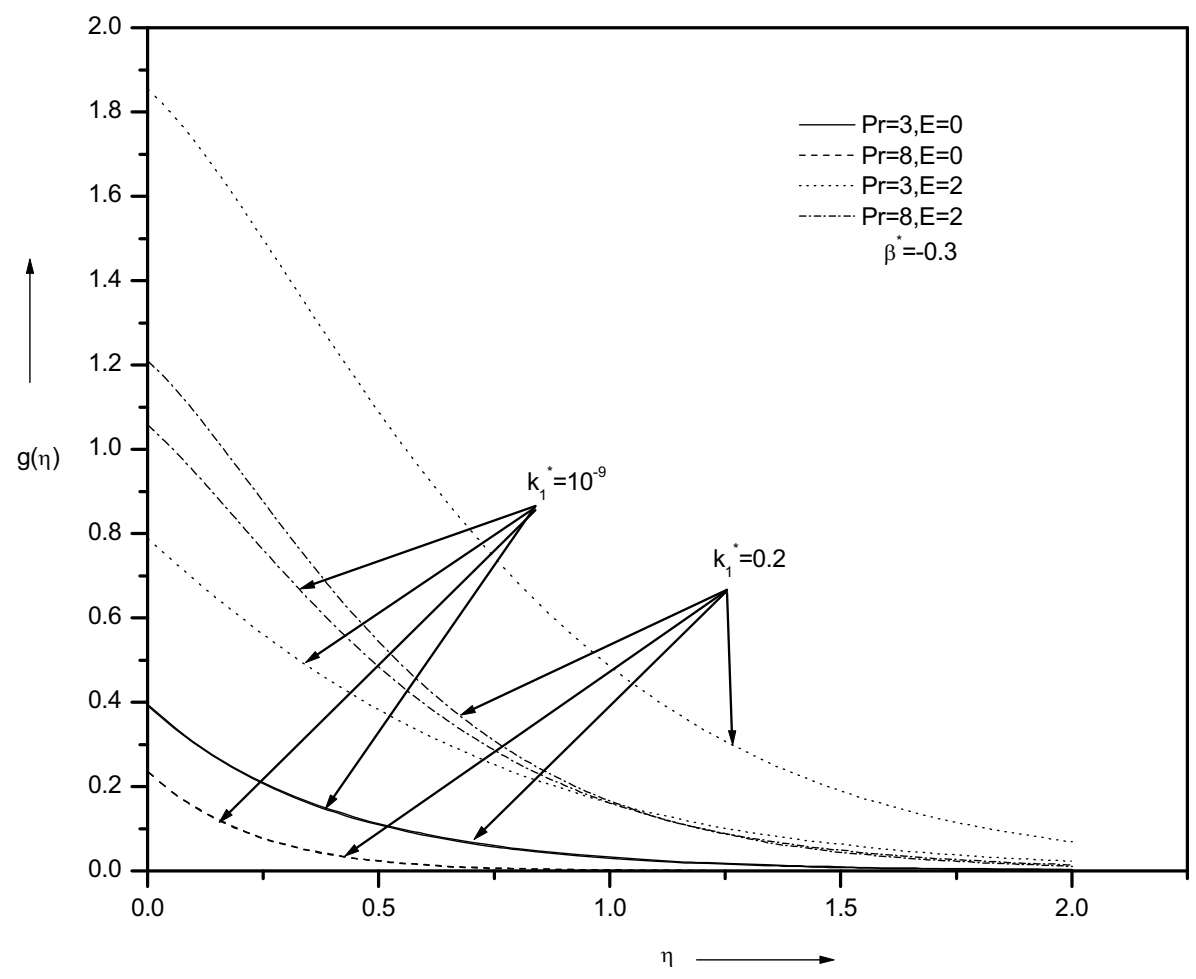

Fig.6b. Dimensionless temperature profile $g(\eta)$ for various values of Prandtl number Pr and Eckert number E in PEHF case when $\beta^{*}=-0.3$.

\section{Conclusions}

A mathematical problem has been formulated on momentum and heat transfer phenomena in a viscoelastic second order fluid flow over an exponentially stretching impermeable sheet with heat source/sink present in the boundary layer. In the solution procedure highly non-linear partial differential equations are converted into a set of quasi-ordinary differential equations by applying pseudo-similarity transformations. Sequential solutions of the transformed momentum equation are obtained analytically by solving the non-linear Riccati type equation repeatedly. A zero-order solution for the stream function $f(\eta)$ and velocity function $f_{\eta}(\eta)$ are obtained analytically. These solutions are compared with the numerical solution of the problem obtained by employing the Runge-Kutta fourth order method involving shooting and good accuracy has been found near the region close to the boundary. Considering the zero-order solution for $f(\eta)$ the solutions of the heat transfer equation are derived in the form of a confluent hypergeometric function for both cases: (i) Prescribed Exponential Order Surface Temperature (PEST) and (ii) Prescribed Exponential Order Surface Heat Flux (PEHF). Expressions are also obtained for the dimensionless local skin-friction coefficient $C_{f}$, temperature gradient in the PEST case and surface temperature in the PEHF case. The derived solutions involve an exponential dependence of the stretching velocity, prescribed surface temperature and prescribed boundary heat flux on the flow directional coordinate.

The important findings of the graphical analysis of the results of the present problem are as follows.

1. Zero-order analytical solutions of the stream function $f(\eta)$ and velocity function $f_{\eta}(\eta)$ have good accuracy near the region very close to the boundary sheet. 
2. The effect of increasing the values of the local viscoelastic parameter $k_{l}^{*}$ decreases the velocity throughout the boundary layer.

3. The effect of increasing the values of the local viscoelastic parameter $k_{l}^{*}$ decreases the local skinfriction parameter $C_{f}$ and the effect of the local Reynolds number Re also decreases the skin-friction parameter $C_{f}$.

4. The effect of increasing the values of the Prandtl number Pr decreases temperature distribution in the flow region in the absence of viscous dissipation. Whereas, the effect of increasing the values of the local viscoelastic parameter $k_{l}^{*}$ increases the temperature distribution in the flow region.

5. In the presence of viscous dissipation $(\mathrm{E} \neq 0)$ and local heat source $\left(\beta^{*}>0\right)$ there may be a higher temperature distribution near the boundary for higher values of the Prandtl number. This region of higher temperature distribution for higher values of the Prandtl number Pr decreases with the increase of the local viscoelastic parameter $k_{l}^{*}$.

6. The rate of heat transfer from the stretching sheet to the fluid can be controlled by suitably choosing the values of the Prandtl number Pr and local Eckert number E, local viscioelastic parameter $k_{l}^{*}$ and local heat source/ sink parameter $\beta^{*}$.

7. The wall temperature gradient $-\theta_{\eta}(0)$ attains a minimum value with the decrease of the Prandtl number Pr and increase of the local viscoelastic parameter $k_{l}^{*}$ and local Eckert number $\mathrm{E}$ in the presence of a heat/source.

8. The surface temperature distribution can be affected by changing the values of the Prandtl number, local Eckert number, local viscoelastic parameter and local source/sink parameter.

9. The limiting cases of the results of this paper when $k_{l}^{*}=0$ and $\beta^{*}=0$ are in excellent agreement with the results of Elbashbeshy (2001) in the absence of suction.

\section{Acknowledgement}

This work is supported by the University Grants Commission, New-Delhi, under Major Research Project (Grant No39-59/2010 (SR)).

\section{Nomenclature}

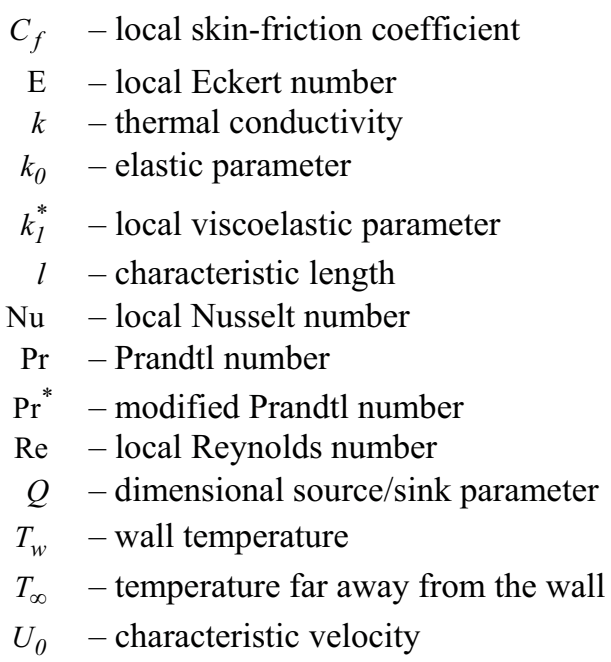


$U_{w} \quad$ - stretching velocity at the wall

$u, v$ - velocity components

$v_{0}, T_{0}$ - temperature parameters in PEST case

$v_{1}, T_{1}$ - temperature parameters in PEHF case

$\beta^{*} \quad-$ non-dimensional local source/sink parameter

$\eta \quad$ - pseudo-similarity variable

$\mu \quad-$ limiting viscosity at small rate of shear

\section{References}

Abramowitz M. and Stegun L.A. (1972): Handbook of Mathematical Functions. - National Bureau of Standards/Amer Math Soc. 55, Providence, RI.

Ali M.E. (1995): On thermal boundary layer on a power law stretched surface with suction or injection. - Int. J. Heat Mass Flow, vol.16, pp.280-290.

Andersson H.I. (1992): MHD flow of a viscoelastic fluid past a stretching surface. - Acta. Mech., vol.95, pp.227-230.

Chang W.D. (1989): The non-uniqueness of the flow of a viscoelastic fluid over a stretching sheet. - Quart. Appl. Math., vol.47, 2, pp.365-366.

Char M.I. (1994): Heat and mass transfer in a hydromagnetic flow of viscoelastic fluid over a stretching sheet. - J. of Math. Anal. Appl., vol.186, pp.674-689.

Coleman B.D. and Noll W. (1990): An approximation theorem for functionals with applications in continuum mechanics. - Arch. Rat. Mech. Anal., vol.6, pp.355.

Cortell R. (1994): Similarity solutions for flow and heat transfer of a viscoelastic fluid over a stretching sheet. - Int. J. Non-Linear Mechanics., vol.29 2, pp.155-161.

Cortell R. (2005): Flow and heat transfer of a fluid through porous medium over a stretching sheet with internal heat generation/absorption and suction/blowing. - Fluid. Dyn. Res., vol.37, pp.231-245.

Cortell R. (2006): A note on flow and heat transfer of a viscoelastic liquid over a stretching sheet. - Int. J. Non. Lin. Mech., vol.41, pp.78-85.

Crane L.J (1970): Flow past a stretching plate. - ZAMP., vol.21, pp.645-647.

Dandapat B.S and Gupta A.S. (1989): Flow and heat transfer in a viscoelastic fluid over a stretching sheet. - Int. J. Non-Linear Mech., vol.24, 3, pp.215-219.

Dandapat B.S., Holmedal L.E. and Andersson H.I. (1994): Stability of a viscoelastic fluid over a stretching sheet. Arch. Mech., vol.46 (6), pp.829-838.

Elbashbeshy E.M.A. (2001): Heat transfer over an exponentially stretching continuous surface with suction. - Arch. Mech., vol.53, (6), pp.643-651.

Fosdick R.L. and Rajagopal K.R. (1979): Anomalous features in the model of second order fluids. - Arch. Ration. Mech. Anal., vol.70, pp.145-152.

Gupta P.S and Gupta A.S. (1977): Heat and mass transfer on a stretching sheet with suction or blowing. - Canad. J. of Chem. Engg., vol.55, pp.744-746.

Khan S.K. (?): Sequential similarity solution of boundary layer equation arising in viscoelastic fluid flow over an exponential stretching sheet. - Proceedings of the International Symposium on Recent Advances in Fluid Mechanics (Tata Mc.Graw-Hill Publication), pp.225-233.

Khan S.K. and Sanjayanand E. (2004): Viscoelastic boundary layer MHD flow through a porous medium over a porous quadratic stretching sheet. - Arch. Mech., vol.56 (3), pp.191-206

Khan S.K. and Sanjayanand. E. (2005): Viscoelastic boundary layer flow and heat transfer over an exponential stretching sheet. - Int. J. Heat and Mass Transfer., vol.48, pp.1534-1542. 
Kumaran V. and Ramanaiah G. (1996): A note on the flow over a stretching sheet. - Acta Mech., vol.116, pp.229-233.

Lawrence P.S. and Rao B.N. (1992): Heat transfer in the flow of viscoelastic fluid over a stretching sheet. - Acta. Mech. vol.93, pp.53-61.

Mahaparta T.R. and Gupta A.S. (2004): Stagnation-point flow of a viscoelastic fluid toward a stretching surface. Inter. J. of Non-Linear Mech., vol.39, pp.811-820.

Prasad K.V., Subhas A.M. and Khan S.K. (2000): Momentum and heat transfer in viscoelastic fluid flow in a porous medium over a non-isothermal stretching sheet. - International Journal of Numerical Methods for Heat and Fluid Flow., vol. 10 (8), pp.786-801.

Prasad K.V., Subhas A.M., Khan S.K. and Datti P.S. (2002), Non-Dracy forced convective heat transfer in a viscoelastic fluid flow over a non-isothermal stretching sheet. - J. Porous Media., vol.5(1), pp.41-47.

Rajagopal K.R., Na T.Y. and Gupta A.S. (1984): Flow of viscoelastic fluid over a stretching sheet. - Rheol. Acta., vol.23, pp.213-215.

Rajagopal K.R., Na T.Y. and Gupta A.S. (1987): A non similar boundary layer on a stretching sheet in a nonNewtonian fluid with uniform free stream. - J. Math. Phy. Sc., vol.21, 2, pp.189-200.

Rollins D. and Vajravelu K. (1991): Heat transfer in a second order fluid over a continuous stretching surface. - Acta. Mech., vol.89, pp.167-178.

Sakiadis B.C. (1961): Boundary layer behaviour on continuous solid surfaces: 1. Boundary layer equations for two dimensional and axisymmetric flows. - A.I.Ch. E.J., vol.7, pp.26-28.

Sanjayanand E. and Khan S.K. (2006): On heat and mass transfer in a viscoelastic boundary layer flow over an exponentially stretching sheet. - Int. J. Therm. Sci., vol.45, pp.819-828.

Troy W.C., Overman E.A.H., Ermentrout G.B. and Keener J.P. (1987): Uniqueness of flow of a second order fluid past a stretching sheet. - Quart. Appl. Math., vol.44, 4, pp.753 -755.

Vajravelu K. (1994): Flow and heat transfer in a saturated porous medium over a stretching surface. - ZAMM, vol.74, 12, pp.605-614.

Received: June 5, 2012

Revised: May 28, 2013 